\title{
Low Diastolic Blood Pressure and Cognitive Decline in Korean Elderly People: The Korean Longitudinal Study on Cognitive Aging and Dementia
}

\author{
Dongyun Lee, ${ }^{1,2}$, Bong-Jo Kim ${ }^{2,3} \bowtie$, Ji Won Han ${ }^{4}$, Tae Hui Kim5, Kyung Phil Kwak ${ }^{6}$, Kayoung Kim \\ Shin Gyeom Kim${ }^{8}$, Jeong Lan Kim ${ }^{9}$, Tae Hyun Kim${ }^{4}$, Seok Woo Moon ${ }^{10}$, Jae Young Park, \\ Joon Hyuk Park ${ }^{11}$, Seonjeong Byun ${ }^{4}$, Seung Wan Suh ${ }^{4}$, Ji Young Seo ${ }^{1}$, Yoonseop So ${ }^{4}$, \\ Seung-Ho Ryu ${ }^{12}$, Jong Chul Youn ${ }^{13}$, Kyoung Hwan Lee ${ }^{14}$, Dong Young Lee ${ }^{15,16}$, Dong Woo Lee ${ }^{17}$, \\ Seok Bum Lee ${ }^{18}$, Jung Jae Lee ${ }^{18}$, Ju Ri Lee ${ }^{4}$, Hyeon Jeong ${ }^{4}$, Hyun-Ghang Jeong ${ }^{19}$, \\ Jin Hyeong Jhoo ${ }^{20}$, Kyuhee $\mathrm{Han}^{4}$, Jong Woo Hong ${ }^{4}$, Jong Bin Bae${ }^{4}$, and Ki Woong Kim ${ }^{4,16,21}$ \\ 1'Department of Psychiatry, Gyeongsang National University Changwon Hospital, Changwon, Republic of Korea \\ ${ }^{2}$ Department of Psychiatry, Gyeongsang National University College of Medicine, Jinju, Republic of Korea \\ ${ }^{3}$ Department of Psychiatry, Gyeongsang National University Hospital, Jinju, Republic of Korea \\ ${ }^{4}$ Department of Neuropsychiatry, Seoul National University Bundang Hospital, Seongnam, Republic of Korea \\ ${ }^{5}$ Department of Psychiatry, Yonsei University Wonju Severance Christian Hospital, Wonju, Republic of Korea \\ ${ }^{6}$ Department of Psychiatry, Dongguk University Gyeongju Hospital, Gyeongju, Republic of Korea \\ ${ }^{7}$ Department of Psychiatry, National Center for Mental Health, Seoul, Republic of Korea \\ ${ }^{8}$ Department of Neuropsychiatry, Soonchunhyang University Bucheon Hospital, Bucheon, Republic of Korea \\ ${ }^{9}$ Department of Psychiatry, School of Medicine, Chungnam National University, Daejeon, Republic of Korea \\ ${ }^{10}$ Department of Psychiatry, School of Medicine, Konkuk University, Konkuk University Chungju Hospital, Chungju, Republic of Korea \\ ${ }^{11}$ Department of Neuropsychiatry, Jeju National University Hospital, Jeju, Republic of Korea \\ ${ }^{12}$ Department of Psychiatry, School of Medicine, Konkuk University, Konkuk University Medical Center, Seoul, Republic of Korea \\ ${ }^{13}$ Department of Neuropsychiatry, Kyunggi Provincial Hospital for the Elderly, Yongin, Republic of Korea \\ ${ }^{14}$ Department of Psychiatry, Bongseng Memorial Hospital, Busan, Republic of Korea \\ ${ }^{15}$ Department of Neuropsychiatry, Seoul National University Hospital, Seoul, Republic of Korea \\ ${ }^{16}$ Department of Psychiatry, Seoul National University College of Medicine, Seoul, Republic of Korea \\ ${ }^{17}$ Department of Neuropsychiatry, Inje University Sanggye Paik Hospital, Seoul, Republic of Korea \\ ${ }^{18}$ Department of Psychiatry, Dankook University Hospital, Cheonan, Republic of Korea \\ ${ }^{19}$ Department of Psychiatry, Korea University Guro Hospital, Korea University College of Medicine, Seoul, Republic of Korea \\ ${ }^{20}$ Department of Psychiatry, Kangwon National University School of Medicine, Chuncheon, Republic of Korea \\ ${ }^{21}$ Department of Brain and Cognitive Science, Seoul National University College of Natural Sciences, Seoul, Republic of Korea
}

Objective Cardiovascular diseases are representative risk factors for the onset of cognitive decline. The purpose of this study was to confirm the relationship between diastolic blood pressure and cognitive function in elderly people in Korea.

Methods Data from subjects who were enrolled in the prospective Korean Longitudinal Study on Cognitive Aging and Dementia were used in this study. Data from 701 subjects whose diastolic blood pressure range did not change ( $\leq 79 \mathrm{~mm} \mathrm{Hg}$ or $\geq 80 \mathrm{~mm} \mathrm{Hg}$ ) over 2 years were analyzed. To analyze the differences in cognitive function between the groups at the 2-year follow-up, an analysis of covariance was performed with covariates, which were significantly different between the two groups, and the baseline cognitive function.

Results Significant differences were observed between the two groups, and the mean scores on the constructional praxis $\left(\eta^{2}=0.010\right)$ and word list recall tests $\left(\eta^{2}=0.018\right)$ in the diastolic blood pressure $\geq 80 \mathrm{~mm} \mathrm{Hg}$ group were higher than those in the diastolic blood pressure $\leq 79 \mathrm{~mm} \mathrm{Hg}$ group at the 2-year follow-up.

Conclusion These results indicate that maintaining a DBP below $79 \mathrm{~mm} \mathrm{Hg}$ presents a greater risk of cognitive decline in Korean elderly people.

Psychiatry Investig 2020;17(1):21-28

Key Words Cognition, Diastolic blood pressure, Senility.

Received: April 29, 2019 Revised: June 27, 2019 Accepted: August 26, 2019

$\triangle$ Correspondence: Bong-Jo Kim, MD, PhD

Department of Psychiatry, College of Medicine, Gyeongsang National University, 15 Jinju-daero 816beon-gil, Jinju 52727, Republic of Korea

Tel: +82-55-750-8084, Fax: +82-55-759-0003, E-mail: bjkim@gnu.ac.kr

(c) This is an Open Access article distributed under the terms of the Creative Commons Attribution Non-Commercial License (https://creativecommons.org/licenses/by-nc/4.0) which permits unrestricted non-commercial use, distribution, and reproduction in any medium, provided the original work is properly cited 


\section{INTRODUCTION}

Dementia is increasing worldwide, and more than 130 million people are expected to suffer from dementia by 2050 if the current trend continues. ${ }^{1}$ In Korea, population aging is increasing, and dementia and cognitive decline in elderly people are of increasing interest. ${ }^{2}$ In a 2014 meta-analysis of the Korean population, the prevalence of dementia and cognitive decline in individuals aged over 65 years was $9.2 \%$, and this prevalence is higher than that in other Asian countries. ${ }^{3} \mathrm{Be}-$ cause health-related problems in elderly people who suffer from cognitive decline and dementia decrease the quality of life of these patients and increase the physical and economic burden on society and caregivers, experts from various fields have increasingly focused on dementia. ${ }^{3,4}$ Because there is no disease-modifying treatment for dementia, the early detection and prevention of dementia and cognitive decline are the most effective strategies. Therefore, identifying the risk factors of cognitive decline is of important clinical significance. Cardiovascular disease is a major risk factor for cognitive decline in elderly people; ${ }^{5-7}$ a recent systematic review found that the prevalence of dementia could decrease by $35 \%$ if modifiable risk factors were corrected. Among the modifiable risk factors, hypertension was the greatest contributor. ${ }^{1}$

Proper blood pressure control is necessary for maintaining cognitive function, especially in elderly people, because hypertension is a major cardiovascular disease that can affect cognitive decline and is one of the pathological mechanisms of Alzheimer disease (AD) and vascular dementia (VaD). ${ }^{8}$ In a prospective cohort study involving elderly patients aged over 70 years who were followed up for 15 years, the risk of $\mathrm{AD}$ and $\mathrm{VaD}$ was increased due to hypertension-induced cardiovascular disease. ${ }^{6}$ A recent meta-analysis of cognitive function and blood pressure in elderly people found that hypertension could reduce global cognitive function. ${ }^{9}$ Furthermore, a previous review showed that high blood pressure in elderly patients aged over 70 years could cause cognitive impairment in areas, such as attention, language fluency, and mental behavior, by causing subcortical white matter lesions in the brain. ${ }^{5}$ However, previous studies have mainly been interested in systolic blood pressure (SBP), and the results of previous studies examining the relationship between diastolic blood pressure (DBP) and cognitive function are controversial. . $^{5,9}$

A previous systematic study suggested that the risk of dementia decreases as DBP increases in late life. ${ }^{10}$ Additionally, a prospective cohort study found that DBP decreases during the years prior to developing dementia. ${ }^{6}$ In contrast, another prospective cohort study showed that low DBP in middle age (age of 50-60 years) was associated with good cognitive function performance in areas, such as verbal attention, fluency, psychomotor speed, and shifting capacity, 20 years later during the later life stages. ${ }^{5}$ Additionally, a recent meta-analysis showed that high DBP is a major predictor of cognitive decline in areas, such as episodic memory and memory recall, and the deterioration of executive function. ${ }^{9}$ The above-mentioned results show that the association between DBP and cognitive function is inconsistent. These inconsistent results might be due to antihypertensive medication use among elderly people with hypertension. ${ }^{11-13}$

In previous studies, the relationship between DBP and cognitive function has been inconsistent in elderly people. ${ }^{8,13}$ When evaluating cognitive function decline in elderly people, it is necessary to consider diverse areas, including memory, language, visuospatial function, and executive function, but previous studies have only evaluated limited specific areas. ${ }^{6,13-16}$ The relationship between DBP and cognitive function in elderly people is inconsistent, and factors related to blood pressure and cognitive function could be affected by ethnic genetic influences and regional lifestyles; therefore, the DBP threshold for maintaining cognitive function may differ among subjects. ${ }^{12,17,18}$ DBP might have a greater effect on cerebral perfusion than SBP, and DBP is related to cognitive decline more than SBP in elderly people. ${ }^{19,20}$ However, to date, no prospective study has examined the relationship between DBP and cognitive function in Korean elderly people. Therefore, the purpose of this study was to investigate the relationship between DBP and cognitive function in elderly people using Korean national prospective cohort data while considering diverse sociodemographic characteristics and physical characteristics.

\section{METHODS}

\section{Subjects}

Data from subjects enrolled in the Korean Longitudinal Study on Cognitive Aging and Dementia (KLosCAD) were used in this study. The KLosCAD is a prospective cohort study involving 14 university hospitals and 2 geriatric hospitals. The purpose of the KLosCAD is to propose a standard of normal aging for cognition and create strategies for the early diagnosis and treatment of dementia. The subjects involved in these studies were elderly people aged over 65 years.

The KLosCAD has been ongoing from 2010 to the present, subject enrollment began in 2011, and the study was designed as a population-based, prospective cohort study. The enrolled subjects are evaluated every 2 years, and three follow-up observations have been conducted. ${ }^{2}$ In total, 6818 subjects registered in the KLosCAD completed the 2-year follow-up. Of these subjects, 2759 were excluded based on the following criteria: the presence of definite dementia $(\mathrm{AD}, \mathrm{VaD}$, mixed type, 
Lewy body, Parkinson's disease, fronto-temporal lobe dementia, alcoholic dementia, dementia due to head trauma, and dementia not otherwise specified), stroke, use of antihypertensive medication, and incomplete information. Finally, data from 701 subjects whose DBP range did not change ( $\leq 79$ $\mathrm{mm} \mathrm{Hg}$ or $\geq 80 \mathrm{~mm} \mathrm{Hg}$ ) over 2 years were analyzed in this study. The researchers who participated in this study were thoroughly educated about all evaluations. This study was approved by the Institutional Review Board of Seoul National University Bundang Hospital (IRB number: B-0912-089010), and written informed consent was obtained from each subject or caregiver after a complete explanation of the study was provided.

\section{Measurements}

Subjects enrolled in the KLosCAD were evaluated using clinical assessments and neurocognitive tests. The clinical assessments included sociodemographic characteristics, physical characteristics, and self-report depressive symptoms. Detailed information regarding each assessment is provided below.

\section{Sociodemographic characteristics}

The subject information included age (years), gender, education level (years), exercise time (minutes/week), alcohol consumption (units/week), cigarette smoking (pack/day), and diabetes mellitus (DM) medication use.

\section{Physical characteristics}

After the subjects rested for more than 5 minutes in a seated or reclined position, their blood pressure was measured three times using a manual desk-type mercury sphygmomanometer, and the mean SBP and DBP ( $\mathrm{mm} \mathrm{Hg}$ ) values were calculated. The American College of Cardiology/American Heart Association provided a new guideline for the evaluation, diagnosis, prevention, and treatment of hypertension in 2018. In this guideline, a DBP above $80 \mathrm{~mm} \mathrm{Hg}$ was suggested as the diagnostic criterion for hypertension. ${ }^{21}$ In the Korean medical field, the application of this guideline is under consideration. All subjects were divided into two groups based on the DBP cutoff of $80 \mathrm{~mm} \mathrm{Hg}$ at baseline and the 2-year followup (LOW DBP group: DBP $\leq 79 \mathrm{~mm} \mathrm{Hg}$, HIGH DBP group: DBP $\geq 80 \mathrm{~mm} \mathrm{Hg}$ ).

The body mass index (BMI, $\mathrm{kg} / \mathrm{m}^{2}$ ) was calculated by measuring the height and weight of the subjects who could be directly assessed; otherwise, BMI was determined by self-report.

\section{Self-reported depressive symptoms}

The depressive symptoms of the subjects were assessed by the Geriatric Depression Scale-Korean version (GDS-KR). The
GDS-KR consists of 30 items, 9 of which are reverse scored. The validity and reliability of the GDS-KR were confirmed in a previous study involving Korean elderly people. ${ }^{22}$

\section{Neurocognitive tests}

The cognitive function of the subjects was assessed using the Korean version of the Consortium to Establish a Registry for Alzheimer's Disease (CERAD-K). In 2002, the CERAD-K was standardized for validity and reliability in a study involving Korean elderly people. ${ }^{23}$ The CERAD-K is divided into the following sub-items: J1, Verbal fluency test; J2, Boston Naming Test; J3, Mini Mental Status Exam for Dementia Screening (MMSE-DS); J4, Word list memory test; J5, Constructional praxis test; J6, Word list recall test; J7, Word list recognition test; J8, Constructional recall test; and J9A/B, Trail-making test $\mathrm{A}$ and $\mathrm{B}$.

\section{Statistical analysis}

Student's t-tests were performed to compare the mean values of the continuous variables, such as age, education level, exercise time, alcohol consumption, cigarette smoking, GDSKR score, SBP, DBP, and BMI, between the groups. Chi-square $\left(\chi^{2}\right)$ tests were performed to compare the categorical variables, such as gender and DM medication use. At each evaluation period, Student's t-tests were performed based on the CERAD-K sub-item scores to compare the cognitive function between the two groups. To analyze the differences in cognitive function between the groups at the 2-year followup, an analysis of covariance (ANCOVA) was performed using a GLM with covariates that significantly differed between the two groups and the baseline CERAD-K sub-item scores. The covariates in ANCOVA were the sociodemographic variables and physical characteristics that were significantly different between the groups. All statistical analyses were performed using SPSS Statistics version 21.0 (IBM Corporation, Armonk, NY, USA). Significance was defined as a 2-tailed p-value less than 0.05 .

\section{RESULTS}

\section{Sociodemographic characteristics, physical characteristics, and depressive symptoms}

In total, 392 subjects and 307 subjects were included in the LOW DBP and HIGH DBP groups, respectively. Student's ttests and chi-square tests were performed. The mean age $( \pm \mathrm{SD})$ in the LOW DBP and HIGH DBP groups was 68.9 $( \pm 6.6)$ and $67.6( \pm 5.9)$ years, respectively $(\mathrm{df}=697, \mathrm{p}=0.008)$. The mean educational level in the LOW DBP and HIGH DBP groups was $8.5( \pm 5.0)$ years and $9.4( \pm 5.0)$ years, respectively $(\mathrm{df}=697, \mathrm{p}=0.025)$. At baseline, the mean $\mathrm{SBP}$ value $( \pm \mathrm{SD})$ in 
the LOW DBP and HIGH DBP groups was $116.0( \pm 10.8) \mathrm{mm} \mathrm{Hg}$ and $130.8( \pm 14.0) \mathrm{mm} \mathrm{Hg}$, respectively $(\mathrm{df}=697, \mathrm{p}<0.001)$. At baseline, the mean BMI $( \pm S D)$ in the LOW DBP and HIGH DBP groups was $23.4( \pm 2.9) \mathrm{m}^{2} / \mathrm{kg}$ and $23.9( \pm 2.9) \mathrm{m}^{2} / \mathrm{kg}$, respectively ( $\mathrm{df}=626, \mathrm{p}=0.038$ ). At the 2 -year follow-up, the mean BMI $( \pm \mathrm{SD})$ in the LOW DBP and HIGH DBP groups was $23.04( \pm 3.0) \mathrm{m}^{2} / \mathrm{kg}$ and $23.9( \pm 5.8) \mathrm{m}^{2} / \mathrm{kg}$, respectively $(\mathrm{df}=427, \mathrm{p}=0.010)$. At baseline, the mean GDS-KR score $( \pm \mathrm{SD})$ in the LOW DBP and HIGH DBP groups was $10.0( \pm 6.5)$ and $8.7(\mathrm{SD}= \pm 5.8)$, respectively $(\mathrm{df}=685, \mathrm{p}=0.005)$. At the 2 -year follow-up, the mean GDS-KR score $( \pm S D)$ in the LOW DBP and HIGH DBP groups was $9.5( \pm 6.8)$ and $7.8( \pm 5.7)$, respectively $(\mathrm{df}=685, \mathrm{p}<0.001)$ (Table 1$)$.

\section{Differences in cognitive function between the LOW DBP and HIGH DBP groups at baseline and}

\section{the 2-year follow-up}

At baseline, there were significant differences between the LOW DBP and HIGH DBP groups in CERAD-K sub-items J1, J2, J6, J8, and J9B based on Student's t-tests. The mean scores $( \pm \mathrm{SD})$ on each sub-item in the LOW DBP and HIGH DBP groups, respectively, were as follows: J1, $14.7( \pm 0.2)$ and $15.1( \pm 0.3), \mathrm{t}=-2.2, \mathrm{df}=694, \mathrm{p}=0.026 ; \mathrm{J} 2,10.7( \pm 0.1)$ and 11.4 $( \pm 0.1), \mathrm{t}=-3.3, \mathrm{df}=612, \mathrm{p}=0.001 ; \mathrm{J} 6,4.9( \pm 0.1)$ and $5.3( \pm 0.1)$, $\mathrm{t}=-2.2, \mathrm{df}=694, \mathrm{p}=0.026 ; \mathrm{J} 8,5.7( \pm 0.2)$ and $6.5( \pm 0.2), \mathrm{t}=-3.1$, $\mathrm{df}=694, \mathrm{p}=0.002$; and J9B, $215.9( \pm 5.7)$ and $191.7( \pm 6.2)$, $\mathrm{t}=2.9, \mathrm{df}=685, \mathrm{p}=0.004$ (Table 2).

At the 2-year follow-up, there were significant differences between the LOW DBP and HIGH DBP groups in CERADK sub-items J2, J3, J4, J5, J6, J8, J9A, and J9B based on Student's t-tests. The mean scores $( \pm \mathrm{SD})$ on the sub-items in the LOW DBP and HIGH DBP groups, respectively, were as follows: J2, $11.1( \pm 0.1)$ and $11.8( \pm 0.1), \mathrm{t}=-3.7, \mathrm{df}=609, \mathrm{p}<0.001$; $\mathrm{J} 3,26.1( \pm 0.2)$ and $27.0( \pm 0.2), \mathrm{t}=-3.7, \mathrm{df}=604, \mathrm{p}<0.001 ; \mathrm{J}$, $16.3( \pm 0.2)$ and $17.3( \pm 0.3), \mathrm{t}=-2.8, \mathrm{df}=611, \mathrm{p}=0.006 ; \mathrm{J}, 9.7$ $( \pm 0.1)$ and $10.1( \pm 0.1), \mathrm{t}=-3.4, \mathrm{df}=608, \mathrm{p}=0.001 ; \mathrm{J} 6,5.2( \pm 0.1)$ and $5.9( \pm 0.1), \mathrm{t}=-3.8, \mathrm{df}=610, \mathrm{p}<0.001 ; \mathrm{J} 8,6.2( \pm 0.2)$ and 6.8 $( \pm 0.2), \mathrm{t}=-2.3, \mathrm{df}=605, \mathrm{p}=0.022 ; \mathrm{J} 9 \mathrm{~A}, 75.8( \pm 3.3)$ and 65.4 $( \pm 2.9), \mathrm{t}=2.4, \mathrm{df}=609, \mathrm{p}=0.017$; and J9B, $203.0( \pm 5.9)$ and $182.1( \pm 6.3), \mathrm{t}=2.4, \mathrm{df}=607, \mathrm{p}=0.016$ (Table 2).

\section{Differences in cognitive function between the LOW DBP and HIGH DBP groups at the 2-year follow-up after adjusting for baseline cognitive function and covariates}

All ANCOVAs included the following covariates: age, education level, baseline SBP, baseline BMI, baseline GDS score, 2-year follow-up BMI and 2-year follow-up GDS score. There were significant differences between the two groups, and the mean score on CERAD-K sub-items J3, J5, and J6 in the HIGH
Table 1. Sociodemographic characteristics by diastolic blood pressure group for all subjects

\begin{tabular}{|c|c|c|c|c|c|}
\hline & \multicolumn{2}{|c|}{$\begin{array}{c}\text { LOW DBP } \\
(\mathrm{N}=392)\end{array}$} & \multicolumn{2}{|c|}{$\begin{array}{l}\text { HIGH DBP } \\
\quad(\mathrm{N}=307)\end{array}$} & \multirow{2}{*}{$\mathrm{p}$} \\
\hline & $\begin{array}{c}\text { Mean } \\
\text { or } \mathrm{N}\end{array}$ & $\begin{array}{c}\text { SD } \\
\text { or } \%\end{array}$ & $\begin{array}{c}\text { Mean } \\
\text { or } \mathrm{N}\end{array}$ & $\begin{array}{c}\mathrm{SD} \\
\text { or } \% \\
\end{array}$ & \\
\hline \multicolumn{6}{|l|}{ Age (years)* } \\
\hline Baseline & 68.9 & 6.6 & 67.6 & 5.9 & $0.008^{\ddagger}$ \\
\hline \multicolumn{6}{|l|}{ Sex $(\text { female })^{\dagger}$} \\
\hline Baseline & 203 & 51.8 & 151 & 49.2 & $0.542^{\S}$ \\
\hline \multicolumn{6}{|c|}{ Education level (years)* } \\
\hline Baseline & 8.5 & 5.0 & 9.4 & 5.0 & $0.025^{\ddagger}$ \\
\hline \multicolumn{6}{|c|}{ Exercise time (minutes/week)* } \\
\hline Baseline & 72.5 & 170.3 & 81.0 & 175.5 & $0.518^{\ddagger}$ \\
\hline Follow-up & 164.4 & 222.7 & 183.1 & 250.1 & $0.300^{\ddagger}$ \\
\hline \multicolumn{6}{|c|}{ Alcohol consumption (units/week)* } \\
\hline Baseline & 4.1 & 12.9 & 5.9 & 14.3 & $0.091^{\ddagger}$ \\
\hline Follow-up & 3.4 & 17.3 & 4.5 & 11.4 & $0.318^{\ddagger}$ \\
\hline \multicolumn{6}{|c|}{ Cigarette smoking (pack/day)* } \\
\hline Baseline & 0.1 & 0.4 & 0.1 & 0.3 & $0.629^{\ddagger}$ \\
\hline Follow-up & 0.1 & 0.3 & 0.1 & 0.3 & $0.624^{\ddagger}$ \\
\hline \multicolumn{6}{|c|}{ Systolic blood pressure $(\mathrm{mm} \mathrm{Hg}) *$} \\
\hline Baseline & 116.0 & 10.8 & 130.8 & 14.0 & $<0.001^{\ddagger}$ \\
\hline Follow-up & 124.5 & 15.1 & 129.1 & 13.0 & $0.624^{\ddagger}$ \\
\hline \multicolumn{6}{|l|}{$\operatorname{BMI}\left(\mathrm{kg} / \mathrm{m}^{2}\right)^{*}$} \\
\hline Baseline & 23.4 & 2.9 & 23.8 & 2.9 & $0.038^{\ddagger}$ \\
\hline Follow-up & 23.0 & 3.0 & 23.9 & 5.8 & $0.010^{\ddagger}$ \\
\hline \multicolumn{6}{|c|}{ Geriatric depression scale* } \\
\hline Baseline & 10.0 & 6.5 & 8.7 & 5.8 & $0.005^{\ddagger}$ \\
\hline Follow-up & 9.5 & 6.8 & 7.8 & 5.7 & $<0.001^{\ddagger}$ \\
\hline \multicolumn{6}{|c|}{ Diabetes mellitus (yes) ${ }^{\dagger}$} \\
\hline Baseline & 9 & 2.3 & 6 & 2.0 & $0.486^{\S}$ \\
\hline Follow-up & 10 & 2.6 & 6 & 2.0 & $0.398^{\S}$ \\
\hline
\end{tabular}

${ }^{*}$ mean $\pm \mathrm{SD},{ }^{\dagger} \mathrm{N}(\%),{ }^{\ddagger} \mathrm{p}$ values from Student's t-test, ${ }^{\S} \mathrm{p}$ values from chi-square test. LOW DBP: diastolic blood pressure $\leq 79 \mathrm{~mm} \mathrm{Hg}$ group, HIGH DBP: diastolic blood pressure $\geq 80 \mathrm{~mm} \mathrm{Hg}$ group, $\mathrm{N}$ : number, SD: standard deviation

group was higher than that in the LOW DBP group at the 2year follow-up as determined by an ANCOVA [J3, F=7.474, $\mathrm{df}=(1,570), \mathrm{p}=0.006, \eta^{2}=0.013 ; \mathrm{J} 5, \mathrm{~F}=5.699, \mathrm{df}=(1,570)$, $\mathrm{p}=0.017, \eta^{2}=0.010$; and $\mathrm{J} 6, \mathrm{~F}=10.688, \mathrm{df}=(1,570), \mathrm{p}=0.001$, $\left.\eta^{2}=0.018\right]$. The mean score on CERAD-K sub-items J4 and J7 did not significantly differ between the two groups at the 2-year follow-up [J4, $\mathrm{F}=2.224, \mathrm{df}=(1,571), \mathrm{p}=0.135, \eta^{2}=0.004$; $\left.J 7, F=0.144, d f=(1,571), p=0.704, \eta^{2}<0.001\right]$. Full results are presented in Table 3. 
Table 2. Comparison of cognitive function between diastolic blood pressure groups for all subjects

\begin{tabular}{|c|c|c|c|c|c|c|}
\hline & \multicolumn{2}{|c|}{ LOW DBP } & \multicolumn{2}{|c|}{ HIGH DBP } & \multirow{2}{*}{$\mathrm{t}$} & \multirow{2}{*}{$\mathrm{p}$} \\
\hline & Mean & $\mathrm{SD}$ & Mean & $\mathrm{SD}$ & & \\
\hline \multicolumn{7}{|c|}{ Verbal fluency test } \\
\hline Baseline & 14.4 & 0.2 & 15.1 & 0.3 & -2.2 & $0.026^{*}$ \\
\hline Follow-up & 14.8 & 0.3 & 15.5 & 0.3 & -1.9 & $0.064^{*}$ \\
\hline \multicolumn{7}{|c|}{ Boston naming test } \\
\hline Baseline & 10.7 & 0.1 & 11.4 & 0.1 & -3.3 & $0.001^{\dagger}$ \\
\hline Follow-up & 11.1 & 0.1 & 11.8 & 0.1 & -3.7 & $<0.001^{\dagger}$ \\
\hline \multicolumn{7}{|l|}{ MMSE-DS } \\
\hline Baseline & 26.3 & 0.2 & 26.6 & 0.2 & -1.6 & $0.120^{*}$ \\
\hline Follow-up & 26.1 & 0.2 & 27.0 & 0.2 & -3.7 & $<0.001^{\dagger}$ \\
\hline \multicolumn{7}{|c|}{ Word list memory test } \\
\hline Baseline & 15.7 & 0.2 & 16.2 & 0.2 & -1.6 & $0.119^{*}$ \\
\hline Follow-up & 16.3 & 0.2 & 17.3 & 0.3 & -2.8 & $0.006^{*}$ \\
\hline \multicolumn{7}{|c|}{ Constructional praxis test } \\
\hline Baseline & 9.6 & 0.1 & 9.7 & 0.1 & -0.8 & $0.401^{*}$ \\
\hline Follow-up & 9.7 & 0.1 & 10.1 & 0.1 & -3.4 & $0.001^{\dagger}$ \\
\hline \multicolumn{7}{|c|}{ Word list recall test } \\
\hline Baseline & 4.9 & 0.1 & 5.3 & 0.1 & -2.2 & $0.026^{*}$ \\
\hline Follow-up & 5.2 & 0.1 & 5.9 & 0.1 & -3.8 & $<0.001^{*}$ \\
\hline \multicolumn{7}{|c|}{ Word list recognition test } \\
\hline Baseline & 8.6 & 0.1 & 8.8 & 0.1 & -1.6 & $0.106^{*}$ \\
\hline Follow-up & 8.7 & 0.1 & 9.0 & 0.1 & -1.8 & $0.078^{\dagger}$ \\
\hline \multicolumn{7}{|c|}{ Constructional recall test } \\
\hline Baseline & 5.7 & 0.2 & 6.5 & 0.2 & -3.1 & $0.002^{*}$ \\
\hline Follow-up & 6.2 & 0.2 & 6.8 & 0.2 & -2.3 & $0.022^{\dagger}$ \\
\hline \multicolumn{7}{|c|}{ Trail-making test A } \\
\hline Baseline & 80.3 & 3.2 & 73.9 & 3.7 & 1.3 & $0.188^{*}$ \\
\hline Follow-up & 75.8 & 3.3 & 65.4 & 2.9 & 2.4 & $0.017^{*}$ \\
\hline \multicolumn{7}{|c|}{ Trail-making test B } \\
\hline Baseline & 215.9 & 5.7 & 191.7 & 6.2 & 2.9 & $0.004^{*}$ \\
\hline Follow-up & 203.0 & 5.9 & 182.1 & 6.3 & 2.4 & $0.016^{*}$ \\
\hline
\end{tabular}

${ }^{*} \mathrm{p}$ values from Student's t-test with homogeneity of variance assumption, ${ }^{\dagger} \mathrm{p}$ values from Student's t-test without homogeneity of variance assumption. LOW DBP: diastolic blood pressure $\leq 79$ $\mathrm{mm} \mathrm{Hg}$ group, HIGH DBP: diastolic blood pressure $\geq 80 \mathrm{~mm} \mathrm{Hg}$ group

\section{DISCUSSION}

This study investigated the relationship between DBP and cognitive function changes in Korean elderly people using data from a national prospective cohort. The main outcomes of this study are that the global cognitive function (J3), visuospatial function (J5), and memory recall (J6) in the LOW DBP group was worse than that in the HIGH DBP group at the 2-year follow-up. These results indicate that compared with a DBP above $80 \mathrm{~mm} \mathrm{Hg}$, a DBP below $79 \mathrm{~mm} \mathrm{Hg}$ has a negative effect on cognitive function in elderly people. Previous studies have identified a relationship between SBP and cognitive function; however, a cohort study investigating cognitive decline in elderly people suggested that DBP is involved in cognition more than SBP. ${ }^{19}$ Cognitive function was closely related to blood pressure in elderly people, and these two variables could be affected by various factors; therefore, investigating subjects with similar conditions, such as in this study, is clinically meaningful.

There was no difference in memory recognition (J7) between the LOW DBP and HIGH DBP groups, but the LOW DBP group had lower scores on memory recall (J6) than the HIGH DBP group. A comparative study investigating cognitive function showed that elderly people with a DBP of 70 $\mathrm{mm} \mathrm{Hg}$ have lower cognitive function in the memory recall domain than those with a higher DBP, even though there is no difference in the other cognitive functions. This study suggests that the memory recall impairment is due to hypoxia and decreased oxygen saturation due to a low cardiac ejection fraction. ${ }^{24}$ In a study using positron emission tomography, both memory recall and memory recognition were positively associated with blood flow (activation) in the prefrontal lobe. However, only memory recall was related to subcortical white matter (SWM), which includes the cerebello-frontal pathway, i.e., the cingulate cortex, globus pallidus, thalamus, and cerebellum; in contrast, these structures are not related to memory recognition. ${ }^{25}$ The above results indicate that memory recall associated with SWM is reduced by cerebral hypoperfusion due to low DBP, while recognition is relatively well maintained in elderly people.

The cognitive domains, including global cognition, memory recall, and visuospatial function, are related to the functional role of SWM in the brain structural domain. Previous studies have shown that lesions in the SWM region can cause cognitive decline. ${ }^{26-29}$ Additionally, a previous review article suggested that SWM lesions decrease the speed of neural transmission and inter-neuronal connectivity. The repeated accumulation of these dysfunctions can lead to cognitive decline. ${ }^{29}$ SWM is easily affected by vascular injury, such as atherosclerosis. An SWM lesion due to early atherosclerosis, such as white matter hyperintensities (WMH) on magnetic resonance imaging, is an asymptomatic lesion; however, this type of lesion has been associated with cognitive decline rather than the pathological level. ${ }^{26,27,30}$ Therefore, the relative decline in cognitive function rather than in the cognitive impairment level in the LOW DBP group compared with the HIGH DBP group may be caused by early atherosclerosis on SWM. 
Table 3. Difference in cognitive function between diastolic blood pressure groups for all subjects at the 2-year follow-up

\begin{tabular}{lcccccc}
\hline & Mean difference & SE & F & df & $\mathrm{p}^{*}$ & $\eta^{2}$ \\
\hline Verbal fluency test & 0.3 & 0.4 & 0.537 & {$[1,572]$} & 0.464 & 0.001 \\
Boston naming test & -0.2 & 0.1 & 1.702 & {$[1,571]$} & 0.193 & 0.003 \\
MMSE-DS & -0.6 & 0.2 & 7.474 & {$[1,570]$} & 0.006 & 0.013 \\
Word list memory test & -0.4 & 0.3 & 2.244 & {$[1,571]$} & 0.135 & 0.004 \\
Constructional praxis test & -0.3 & 0.1 & 5.699 & {$[1,570]$} & 0.017 & 0.010 \\
Word list recall test & -0.5 & 0.2 & 10.688 & {$[1,570]$} & 0.001 & 0.018 \\
Word list recognition test & -0.1 & 0.1 & 0.144 & {$[1,571]$} & 0.704 & $<0.001$ \\
Constructional recall test & 0.1 & 0.2 & 0.125 & {$[1,570]$} & 0.723 & $<0.001$ \\
Trail-making test A & 1.3 & 2.8 & 0.201 & {$[1,570]$} & 0.654 & $<0.001$ \\
Trail-making test B & 2.8 & 7.0 & 0.166 & {$[1,566]$} & 0.684 & $<0.001$ \\
\hline
\end{tabular}

${ }^{*}$ p values from analysis of covariance. SE: standard error, df: degrees of freedom, $\eta^{2}$ (eta squared): effect size

The intima-media thickness of the vessels reflects the degree of atherosclerosis. ${ }^{31}$ In a cohort study involving elderly people aged 70 years and older, the intima-media thickness in the subjects with low DBP (70-79 mm Hg) was thicker than that in those with high DBP; ${ }^{32}$ additionally, in a study investigating the cardiovascular health of elderly people aged 65 years and older, the intima-media thickness of the common carotid artery was thicker in the subjects with lower DBP. ${ }^{31} \mathrm{WMH}$ due to atherosclerosis is indicative of cerebral small vessel diseases, which are related to cognitive decline, and WMH increases the risk of $\mathrm{VaD}$ and $\mathrm{AD} .^{27}$ The relationship between low DBP and the relative decline in the cognitive domain found in the main results of this study may be related to cerebral hypoperfusion due to vascular changes, such as atherosclerosis, in elderly people.

A 5-year follow-up study examining local cerebral blood flow in healthy elderly subjects found that elderly people who showed cognitive function decline after 5 years had a greater reduction in white matter perfusion. ${ }^{33}$ When low DBP is maintained in elderly people, arterial stiffness and atherosclerosis are increased, resulting in poor cerebral perfusion and oxidative stress. ${ }^{19,34,35}$ Cerebral hypoperfusion causes vascular changes, such as endothelial cells and basal membrane damage in blood vessels. Vascular permeability is increased by a damaged blood-brain barrier, resulting in the accumulation of $A \beta$ protein and $\beta$-amyloid protein in the cerebral parenchyma; this accumulation promotes free radical production and causes neuronal cell death. ${ }^{34,35}$ The results of this study, which showed that the visuospatial function and memory recall related to the vascular cognitive domain in the LOW DBP group were lower than those in the HIGH DBP group, suggest that the decrease in cognitive function is due to cerebral hypoperfusion, which is related to atherosclerosis due to low DBP. However, further studies are needed because atherosclerosis could not be confirmed in the subjects by imaging or hematology in this study.

In contrast, several previous studies have reported that high blood pressure is associated with cognitive decline. In a crosssectional study using data from the National Health and Nutrition Examination Survey of United States, the relationship between SBP and cognitive function decline, which was evaluated using the serial digit learning test for the age range from 20 to 50 years, changed with age. ${ }^{36}$ These results indicate that high SBP could predict cognitive decline in adults in their 20s to 30 s, but the relationship was not significant in adults aged 40-50. ${ }^{36}$ A prospective cohort study that followed subjects for 15 years found that patients who had hypertension for more than 10 years had a higher risk of dementia in later life. ${ }^{37}$ In a recent systematic review of dementia onset, hypertension occurring in middle age between the ages of 45 and 65 years was a risk factor for dementia in later life. ${ }^{1}$ Previous studies have indicated that the relationship between cognitive decline and high blood pressure depends on the duration of prolonged exposure to hypertension. High blood pressure that occurs at a relatively young age has been associated with decreased cognitive function in later life. There may be differences between the results reported in previous studies and our results because of the relatively short follow-up period of 2 years. Therefore, since the cohort study is ongoing, it is necessary to pay close attention to the results of the data analysis after the long-term follow-up observation.

There are several limitations in this study. First, the results of the paired t-tests showed that the CERAD-K sub-item scores increased from baseline to the 2-year follow-up. In previous prospective studies examining cognitive function in elderly people, if the same cognitive function evaluation was performed within 2 years, the score could increase due to ceiling (scale attenuation) and learning effects. ${ }^{38,39}$ However, in this study, because we analyzed the relative difference in cognitive function changes between the two groups according to DBP, 
the results were significant. Second, the main results of this study had a small effect size $\left(\eta^{2}=0.001\right)$. However, as noted in a previous study, the small effect size in studies examining the relationship between blood pressure and cognitive function could be due to the diversity of factors that may affect blood pressure and cognitive function. ${ }^{36}$ This study adjusted for various sociodemographic variables and physical characteristics, and the observed period was relatively short ( 2 years). This cohort study is currently ongoing, and the results of the cohort will be analyzed further. Third, because only 701 of the approximately 6,000 KLosCAD registered patients completed the 2-year follow-up and were analyzed in this study, there may be a risk of bias regarding the group characteristics. This study aimed to investigate the relationship between DBP and cognitive function in elderly people with normal cognitive functioning; patients were excluded if they had dementia, a definite cognitive impairment, stroke, or use of antihypertensive medication or when the DBP changed categories during the 2 years because their data could affect the interpretation of the results. The power analysis results evaluating the appropriate sample size for a repeated design study suggested an explanatory power of $95 \%$ or more when analyzing more than 100 participants. Therefore, the sample size of 701 subjects in this study is sufficient. Fourth, some BMIs were calculated based on self-reported height and weight values; therefore, these BMIs might not be accurate.

Despite these limitations, this study analyzed national representative data from Korean elderly people and is the first to show that lower DBP is a risk factor for cognitive decline in Korean elderly people. The relationship between cognitive function and blood pressure in elderly people can be influenced by age, gender, education, lifestyle factors, such as cigarette smoking and alcohol drinking, the presence of metabolic diseases, such as diabetes, and race. ${ }^{12,13,17}$ Therefore, the results of this study are meaningful because the analyses were conducted after adjusting for various factors that could affect cognitive function and DBP among a population of elderly subjects of a single race in Korea.

In conclusion, a DBP less than $79 \mathrm{~mm} \mathrm{Hg}$ in Korean elderly people aged over 65 years had a negative effect on the maintenance of cognitive function over time. The relationship between blood pressure and cognitive function may vary among races. These results indicate that maintaining a DBP below 79 $\mathrm{mm} \mathrm{Hg}$ presents a greater risk of cognitive decline in Korean elderly people. These results could be a cornerstone for further research investigating the causality between DBP and cognitive function in Korean populations.

\section{Acknowledgments}

This study is currently funded by a grant [grant no. HI09C1379 (A092077)] from the Korean Health Technology R\&D Project, Ministry of Health and Welfare, Republic of Korea.

This study was supported by a grant from the Korean Health Technology R\&D Project, Ministry of Health and Welfare, Republic of Korea [grant no. HI09C1379 (A092077)].

\section{Conflicts of Interest}

The authors have no potential conflicts of interest to disclose.

\section{Author Contributions}

Conceptualization: Dongyun Lee, Bong-Jo Kim. Data curation: Dongyun Lee, Ji Won Han. Formal analysis: Dongyun Lee. Funding acquisition: Ki Woong Kim. Investigation: Bong-Jo Kim, Ji Won Han, Tae Hui Kim, Kyung Phil Kwak, Kayoung Kim, Shin Gyeom Kim, Jeong Lan Kim, Tae Hyun Kim, Seok Woo Moon, Jae Young Park, Joon Hyuk Park, Seonjeong Byun, Seung Wan Suh, Ji Young Seo, Yoonseop So, Seung-Ho Ryu, Jong Chul Youn, Kyoung Hwan Lee, Dong Young Lee, Dong Woo Lee, Seok Bum Lee, Jung Jae Lee, Ju Ri Lee, Hyeon Jeong, Hyun-Ghang Jeong, Jin Hyeong Jhoo, Kyuhee Han, Jong Woo Hong, Jong Bin Bae, Ki Woong Kim. Methodology: Dongyun Lee, Bong-Jo Kim, Ji Won Han, Ki Woong Kim. Supervision: Ji Won Han, Ki Woong Kim. Writing_original draft: Dongyun Lee. Writing_-review \& editing: Dongyun Lee, Bong-Jo Kim.

\section{ORCID iDs}

Bong-Jo Kim

Dongyun Lee

https://orcid.org/0000-0003-2419-7306

https://orcid.org/0000-0002-3977-3663

\section{REFERENCES}

1. Livingston G, Sommerlad A, Orgeta V, Costafreda SG, Huntley J, Ames D, et al. Dementia prevention, intervention, and care. Lancet 2017;390: 2673-2734.

2. Kim TH, Park JH, Lee JJ, Jhoo JH, Kim BJ, Kim JL, et al. Overview of the Korean longitudinal study on cognitive aging and dementia. Alzheimers Dement 2013;9:P626-P627.

3. Kim YJ, Han JW, So YS, Seo JY, Kim KY, Kim KW. Prevalence and trends of dementia in Korea: a systematic review and meta-analysis. J Korean Med Sci 2014;29:903-912.

4. Thomas P, Lalloué F, Preux PM, Hazif-Thomas C, Pariel S, Inscale R, et al. Dementia patients caregivers quality of life: the PIXEL study. Int $J$ Geriatr Psychiatry 2006;21:50-56.

5. Birkenhäger WH, Forette F, Seux M, Wang J, Staessen JA. Blood pressure, cognitive functions, and prevention of dementias in older patients with hypertension. Arch Intern Med 2001;161:152-156.

6. Skoog I, Nilsson L, Persson G, Lernfelt B, Landahl S, Palmertz B, et al. 15-year longitudinal study of blood pressure and dementia. Lancet 1996; 347:1141-1145.

7. Kilander L, Nyman H, Boberg M, Lithell H. The association between low diastolic blood pressure in middle age and cognitive function in old age. A population-based study. Age Ageing 2000;29:243-248.

8. Qiu C, Winblad B, Fratiglioni L. The age-dependent relation of blood pressure to cognitive function and dementia. Lancet Neurol 2005;4:487499.

9. Gifford KA, Badaracco M, Liu D, Tripodis Y, Gentile A, Lu Z, et al. Blood pressure and cognition among older adults: a meta-analysis. Arch Clin Neuropsychol 2013;28:649-664.

10. Power MC, Weuve J, Gagne JJ, McQueen MB, Viswanathan A, Blacker D. The association between blood pressure and incident Alzheimer disease: a systematic review and meta-analysis. Epidemiology 2011;22:646659.

11. Kennelly SP, Lawlor B, Kenny R. Blood pressure and the risk for dementia-a double edged sword. Ageing Res Rev 2009;8:61-70.

12. Liu H, Gao S, Hall KS, Unverzagt FW, Lane KA, Callahan CM, et al. Optimal blood pressure for cognitive function: findings from an elderly Af- 
rican-American cohort study. J Am Soc Geriatr Dent 2013;61:875-881.

13. Birns J, Kalra L. Cognitive function and hypertension. J Hum Hypertens 2009;23:86-96

14. Gorelick PB, Nyenhuis D. Blood pressure and treatment of persons with hypertension as it relates to cognitive outcomes including executive function. J Am Soc Hypertens 2012;6:309-315.

15. Hajjar I, Hart M, Chen YL, Mack W, Milberg W, Chui H, et al. Effect of antihypertensive therapy on cognitive function in early executive cognitive impairment: a double-blind randomized clinical trial. Arch Intern Med 2012;172:442-444.

16. Prince MJ, Bird AS, Blizard RA, Mann AH. Is the cognitive function of older patients affected by antihypertensive treatment? Results from 54 months of the medical research council's treatment trial of hypertension in older adults. BMJ 1996;312:801-805.

17. Kario K, Chen CH, Park S, Park CG, Hoshide S, Cheng HM, et al. Consensus document on improving hypertension management in asian patients, taking into account asian characteristics. Hypertension 2018;71: 375-382.

18. Kimm H, Mok Y, Lee SJ, Lee S, Back JH, Jee SH. The J-curve between diastolic blood pressure and risk of all-cause and cardiovascular death. Korean Circ J 2018;48:36-47.

19. Qiu C, Winblad B, Fastbom J, Fratiglioni L. Combined effects of APOE genotype, blood pressure, and antihypertensive drug use on incident AD. Neurology 2003;61:655-660.

20. Wallace RB, Lemke JH, Morris MC, Goodenberger M, Kohout F, Hinrichs JV. Relationship of free-recall memory to hypertension in the elderly. The Iowa 65+ rural health study. J Clin Epidemiol 1985;38:475-481.

21. Daskalopoulou SS, Rabi DM, Schiffrin EL, Feldman RD, Padwal RS, Tremblay G, et al. Hypertension guidelines in the United States and Canada: are we getting closer? Hypertension 2018;71:976-978.

22. Bae JN, Cho MJ. Development of the Korean version of the Geriatric depression scale and its short form among elderly psychiatric patients. J Psychosom Res 2004;57:297-305.

23. Lee JH, Lee KU, Lee DY, Kim KW, Jhoo JH, Kim JH, et al. Development of the Korean version of the consortium to establish a registry for Alzheimer's disease assessment packet (CERAD-K) clinical and neuropsychological assessment batteries. J Gerontol B Psychol Sci Soc Sci 2002;57:P47-P53.

24. Pressler SJ, Subramanian U, Kareken D, Perkins SM, Gradus-Pizlo I, Sauvé MJ, et al. Cognitive deficits in chronic heart failure. Nurs Res 2010;59:127-139.

25. Cabeza R, Kapur S, Craik FI, McIntosh AR, Houle S, Tulving E. Functional neuroanatomy of recall and recognition: a PET study of episodic memory. J Cogn Neurosci 1997;9:254-265.
26. Sevilla R, Naranjo I, Cuenca J, Rodriguez J, Espuela F. Vascular risk factors and white matter hyperintensities as predictors of progression to dementia in patients with mild cognitive impairment. Curr Alzheimer Res 2018;15:671-678.

27. Prins ND, Scheltens $P$. White matter hyperintensities, cognitive impairment and dementia: an update. Nat Rev Neurol 2015;11:157-165.

28. De Groot JC, De Leeuw FE, Oudkerk M, Van Gijn J, Hofman A, Jolles J, et al. Cerebral white matter lesions and cognitive function: the Rotterdam scan study. Ann Neurol 2000;47:145-151.

29. Gunning-Dixon FM, Raz N. The cognitive correlates of white matter abnormalities in normal aging: a quantitative review. Neuropsychology 2000;14:224-232.

30. Bots ML, Witteman JC, Hofman A, De Jong PT, Grobbee DE. Low diastolic blood pressure and atherosclerosis in elderly subjects: the Rotterdam study. Arch Intern Med 1996;156:843-848.

31. Psaty BM, Furberg CD, Kuller LH, Borhani NO, Rautaharju PM, O'Leary DH, et al. Isolated systolic hypertension and subclinical cardiovascular disease in the elderly: initial findings from the cardiovascular health study. JAMA 1992;268:1287-1291.

32. Elias MF, Wolf PA, D'Agostino RB, Cobb J, White LR. Untreated blood pressure level is inversely related to cognitive functioning: the Framingham study. Am J Epidemiol 1993;138:353-364.

33. Meyer JS, Rauch G, Rauch RA, Haque A. Risk factors for cerebral hypoperfusion, mild cognitive impairment, and dementia. Neurobiol Aging 2000;21:161-169.

34. De la Torre JC. Alzheimer disease as a vascular disorder: nosological evidence. Stroke 2002;33:1152-1162.

35. Mecocci P, Polidori MC, Cherubini A, Ingegni T, Mattioli P, Catani M, et al. Lymphocyte oxidative DNA damage and plasma antioxidants in Alzheimer disease. Arch Neurol 2002;59:794-798.

36. Suhr JA, Stewart JC, France CR. The relationship between blood pressure and cognitive performance in the Third National Health and Nutrition Examination Survey (NHANES III). Psychosom Med 2004;66: 291-297.

37. Elias PK, Elias MF, Robbins MA, Budge MM. Blood pressure-related cognitive decline: does age make a difference? Hypertension 2004;44: 631-636.

38. Ganguli M, Seaberg EC, Ratcliff GG, Belle SH, DeKosky ST. Cognitive stability over 2 years in a rural elderly population: the MoVIES project. Neuroepidemiology 1996;15:42-50.

39. Burkhart CS, Birkner-Binder D, Gagneux A, Berres M, Strebel SP, Monsch AU, et al. Evaluation of a summary score of cognitive performance for use in trials in perioperative and critical care. Dement Geriatr Cogn Dis 2011;31:451-459. 